\title{
EDITORIAL
}

\section{Editorial: Swiss Society for Sports Psychiatry and Psychotherapy SSSPP}

\section{EXERCISE IS MEDICINE}

\section{Claussen Malte Christian}

President and Head of Department Adult Psychiatry and Psychotherapy, Swiss Society for Sports Psychiatry and Psychotherapy SSSPP

Head of Department Sports Psychiatry and Psychotherapy, Department of Psychiatry, Psychotherapy and Psychosomatics, University Hospital of Psychiatry Zurich, Zurich, Switzerland, Private Clinic Wyss AG, Muenchenbuchsee, Switzerland and Psychiatric Services Grisons, Chur, Switzerland

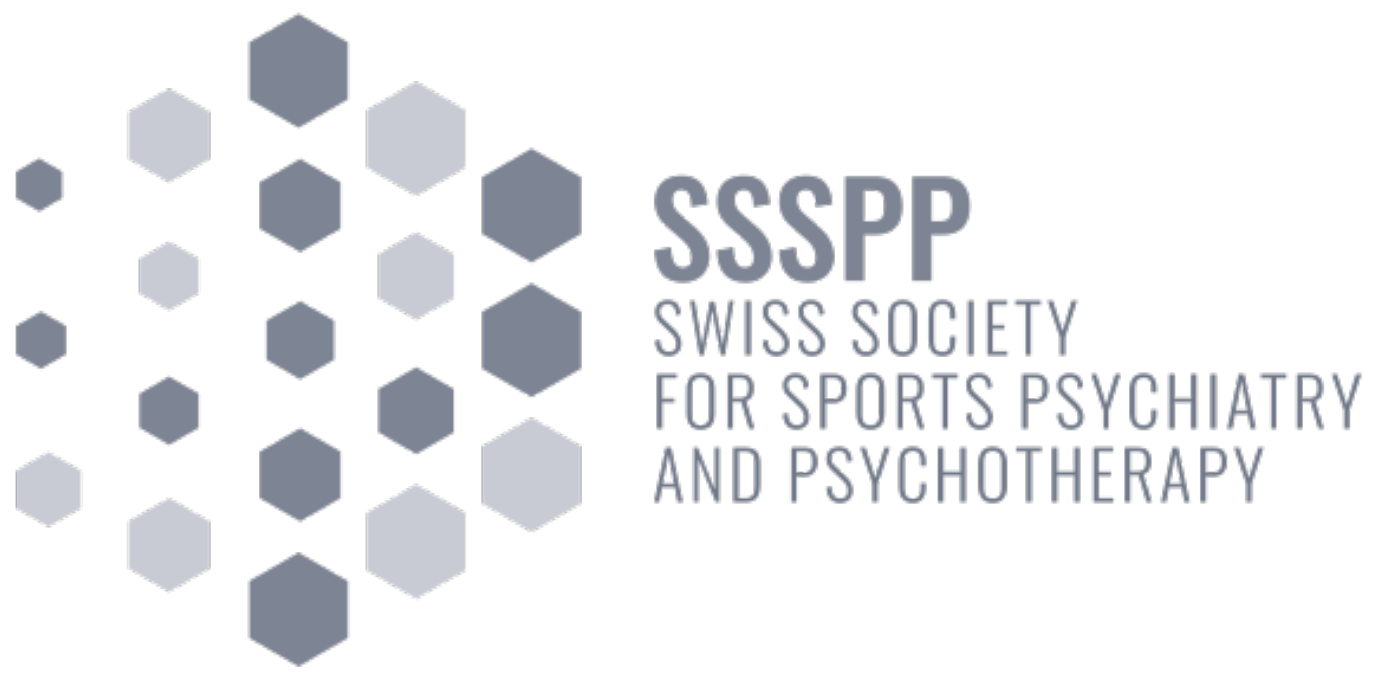

The Swiss Society for Sports Psychiatry and Psychotherapy SSSPP was founded on the grounds of the Private Clinic Wyss in Muenchenbuchsee in the canton of Bern on March 29, 2019. Worldwide, it is the second sports psychiatry and psychotherapy society to be founded after the International Society of Sports Psychiatry ISSP and the first national institution of this sort. The SSSPP aims to implement methods of sports psychiatry and psychotherapy throughout the course of a lifetime within the Swiss population and in competitive sports. This is reflected in the two main task assignments of the SSSPP: mental health in competitive sports and physical activity in the prevention and therapy of mental health diseases. Throughout "the course of a lifetime" demonstrates that the SSSPP would like to promote sports psychiatry 
and psychotherapy for children, adolescents, adults and the elderly in equal measure. This is embodied within the SSSPP board, as there are representatives of psychiatry and psychotherapy for each individual age group as well as a department for research and education.

The SSSPP is a professional sports psychiatry and psychotherapy institution that currently acts as a domain of adult and child/adolescent psychiatry and psychotherapy, but - due to the complex and interdisciplinary nature - would like to be established as a branch of sports medicine. In the view of the SSSPP, sports medicine, sports psychiatry and the SEMS (Sport and Exercise Medicine Switzerland) could be ideally linked by forming an informational exchange platform. The SSSPP is prepared to contribute to this vision in a substantial matter.

The SSSPP would like to advocate interests for sports psychiatrists and psychotherapists and promote the development of sports psychiatry and psychotherapy by including other professionals. Core values include the interdisciplinary informational exchange, e.g. in sports medicine, sports and exercise studies as well as sports psychology, along with bridging the gap to clinical psychiatry or psychotherapy. This can be achieved by forming connections between the subspecialties and their societies, awareness campaigns and publications. Furthermore, SSSPP is a scientific society that supports research and educational work, raises awareness to the prevention of mental health disorders and is committed to destigmatization of the latter. In this edition of Sport \& Exercise Medicine Switzerland, we hope to give insight into research initiatives within sports psychiatry and psychotherapy. Even today, these research initiatives are a result of collaboration between different disciplines, institutions and clinics. The SSSPP supports this exchange by providing a comprehensive website and list of events, which showcases researchers.

Throughout the last few months, the board of SSSPP has developed task assignment policies. These documents will be published in the English language within this issue. First, challenges within the field are described. Second, possible starting points and recommendations to overcome these obstacles are discussed. These policies are open to public debate, as this is essential for the improvement of mental health in competitive sports, as well as for the inclusion of sports, exercise and movement in prevention and therapeutic concepts in mental health disorders.

Systematically conferring information is integral for every subspecialty. Over the course of the last few months, a task force within the SSSPP has collaborated with the German Society for Sports Psychiatry and Psychotherapy (GSSPP) to develop a three-part curriculum in qualifying in sports psychiatry and psychotherapy. This program is one of the first within this field. Part one, "Primary care in sports psychiatry and psychotherapy” enables psychiatrists, psychotherapists and sports physicians to gain insight in this field and to acquire basic knowledge. Part two, "clinical sports psychiatry and psychotherapy" aims to demonstrate the clinical value of sports, exercise and movement, while part three focusses on competitive sports and leads to the "sports psychiatry and psychotherapy" diploma of the SSSPP. In the last article, the SSSPP curriculum of sports psychiatry and psychotherapy will be introduced. It was important for the SSSPP to work with various national and international societies to find a consensus and coherent agreements, as the field of sports psychiatry and psychotherapy continuously evolves. This foundation will facilitate the informational exchange, and so, to further establish the field of sports psychiatry and psychotherapy. Events, symposia, conferences, publications, but also interpersonal exchange will further contribute to this goal. The SSSPP is committed to an interdisciplinary and international collaboration.

Regarding the further development of the Swiss Society for Sports Psychiatry and Psychotherapy, any 
contributions are welcome. The second SSSPP annual conference will take place at the University Hospital of Psychiatry Zurich on January 22-23, 2021. Information on participation and membership as well as news can be found on the official website of the SSSPP, http://www.sgspp.ch.

\section{Corresponding author}

Dr. med. Malte Christian Claussen

President SSSPP

c/o Sports Psychiatry and Psychotherapy,

Department of Psychiatry,

Psychotherapy and Psychosomatics,

University Hospital of Psychiatry Zurich

Lenggstrasse 31, CH-8032 Zurich, Switzerland

E-Mail: malte.claussen@pukzh.ch

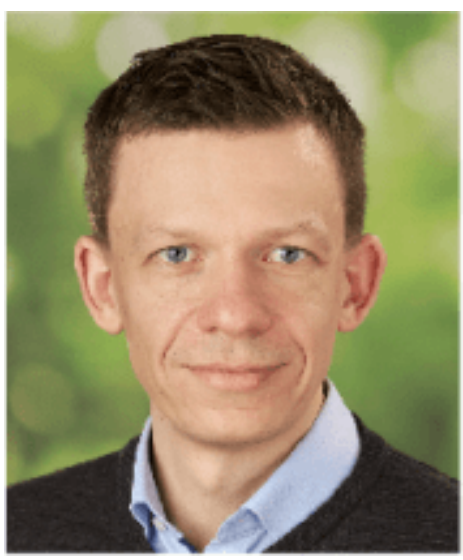

